\title{
Posterior Variant of Alien Limb Syndrome with Sudden Clinical Onset as Self-Hitting Associated with Thalamic Stroke
}

\author{
Mirella Russo $^{a} \quad$ Claudia Carrarini $^{\mathrm{a}} \quad$ Fedele Dono $^{\mathrm{a}} \quad$ Vincenzo Di Stefano $^{\mathrm{a}}$ \\ Maria Vittoria De Angelis ${ }^{a} \quad$ Marco Onofrja $^{\mathrm{a}}$ Stefano L. Sensi ${ }^{\mathrm{a}-\mathrm{C}}$ \\ ${ }^{a}$ Department of Neuroscience, Imaging, and Clinical Sciences, University G. d'Annunzio of \\ Chieti-Pescara, Chieti, Italy; ${ }^{b}$ Behavioral Neurology and Molecular Neurology Units, Center \\ of Excellence on Aging and Translational Medicine - CeSI-MeT, University G. d'Annunzio \\ of Chieti-Pescara, Chieti, Italy; 'Departments of Neurology and Pharmacology, Institute \\ for Mind Impairments and Neurological Disorders - iMIND, University of California, \\ Irvine, CA, USA
}

\section{Keywords}

Alien hand · Posterior variant of alien limb syndrome - Stroke · Cerebrovascular disease . Thalamic stroke

\section{Abstract}

We present a case of sudden postischaemic onset of alien limb syndrome, with unintentional self-injury. Alien limb syndrome is an uncommon neurological disorder featured by uncontrolled and involuntary movements of a limb. Three variants of alien limb syndrome have been described: the anterior, featured by grasping of surrounding objects, the callosal, presenting with intermanual conflict, and the posterior, associated with involuntary levitation of the limb. Our patient suffered from an acute presentation of the posterior variant of the alien limb syndrome, resulting from an isolated thalamic stroke which was documented using 24-h 
computed tomography brain scan. Only one previous case of alien limb syndrome after thalamic infarct has been reported. Our case enhances the possibility that pure thalamic injury may represent a trigger for this condition.

\section{Introduction}

The alien limb syndrome (ALS) was first described in 1908 by Goldstein [1], who defined ALS as a neurological condition characterized by "a stranger and extraneous feeling, typically about one arm, less frequently about one leg, and objective uncontrolled and unintentional movements." To date, three variants of the syndrome are known: the frontal, callosal and posterior variants [2]. The posterior syndrome is typically associated with the presence of unintentional and uncontrolled levitation of the affected limb whereas the frontal and callosal variants exhibit aberrant manipulation or intermanual conflicts [2].

We here present the case of a patient showing an ALS subtype characterized by an abrupt postischaemic onset and the presence of involuntary movements leading to self-injury.

\section{Case Presentation}

On February 2018, an 80-year-old right-handed man was admitted to our neurological ward as a consequence of movement disorders leading to self-inflicted contusive trauma to the left eye. The admission report indicated that, in the morning, the patient had experienced paraesthesia restricted to the left side of his face and the left arm. The patient also showed motor control deficits characterized by spontaneous and unrestrained levitation of the left arm. The patient's daughter indicated that the ocular trauma had resulted from an abrupt uncontrolled movement of the arm that occurred suddenly while the subject was getting dressed. It should be noted that the patient was unaware of the consequences of the movement as he suffered from visual impairment. The abrupt movement resulted in a severe blastlike contusion that eventually led to the surgical removal of the eye. On admission, the patient was vigilant and alert, the neurological examination showed moderate $(+3)$ weakness in the left superior and inferior limbs, mild paresis, of central origin, of the cranial nerve VII, and absence of sensory deficits. The finger-to-nose test revealed the presence of segmental ataxia restricted to the left arm. Neglect was absent. The patient had an uncontrolled, spontaneous levitation of the left arm that persisted even when performing distraction manoeuvres. The patients reported that he felt that the limb was extraneous and disturbing to the point he asked to have the arm tied to the bed. We did not observe paroxysmal limb movements. During the first day of hospitalization, the patient was neuroradiologically examined with a computed tomography (CT) brain scan that was negative for signs of recent or ongoing ischaemic or haemorrhagic events. The CT scan only showed the presence of an old malacic lesion in the right occipitoparietal region. A second CT brain scan, performed $24 \mathrm{~h}$ later (Fig. 1), revealed an ischaemic lesion localized in the right thalamus. The patient showed signs of cognitive impairment when tested with the Mini-Mental State Examination (MMSE raw score: 23/30, 23,4/30 when corrected for age and education). Signs consistent with ALS persisted during the 4-day hospitalization of the patient. An EEG recording was performed on day 3 and showed no signs of epileptic abnormalities. At the 2-month follow-up visit, the patient did not show signs of arm levitation, limb ataxia or sensorimotor deficits. The cognitive impairment persisted (MMSE 23/30, corrected score: 23,4/30). The cognitive deficits mainly were 
delayed recall, mild temporal disorientation, poor verbal fluency, and poor sustained attention. The history of our patient did not reveal the presence of underlying risk factors for small vessel disease (i.e., diabetes, hypertension, hypercholesterolaemia) nor signs of carotid artery stenosis. The patient indicated that he had previously suffered from an episode of "arrhythmia" that he could not further describe nor provide any ECG for. Thus, in agreement with the Italian ISO-SPREAD stroke guidelines [3; Recommendation 5.4.c, 2016], we decided to implant a loop recorder and investigated the possibility of atrial fibrillation that was indeed discovered to be present. We, therefore, believe that atrial fibrillation is the most likely origin of the ischaemia suffered by our patient. Accordingly, anticoagulant therapy with apixaban was started.

\section{Discussion}

The present ALS case is intriguing as the condition showed by the patient had an acute onset and was triggered by an isolated thalamic stroke, an uncommon ALS feature [2, 4-7]. ALS is usually subacute and occurs days or weeks after the appearance of motor deficits or other stroke-related symptoms [2, 8, 9]. To date and to our knowledge, only a few cases of ischaemic posterior ALS variants have been associated with isolated thalamic lesions $[4,5,8$, 10-12] as most instances are due to occlusion of the posterior cerebral artery, mainly in the P1 and P2 segments [13-15]. Partial or complete occlusions of the posterior cerebral artery generally result in infarctions that extend beyond the thalamic territory [16]. However, cases of isolated thalamic infarcts can be found in small vessel disease, sometimes associated with movement disorders [17]. Thus, our case suggests that the thalamus can act as a highly localized trigger for the syndrome. The case supports the notion that a restricted thalamic lesion is sufficient to produce a posterior variant of ALS. A possible mechanism for this distinct thalamic driver involves the occurrence of a "diaschisis effect" derived from an ongoing frontoparietal disconnection $[2,10,18]$. An alternative mechanism may relate to the limb sensory deficits caused by lesions localized within the somatosensory nuclei of the thalamus (ventral posteromedial and ventral posterolateral). This, mechanism is in line with the presence of sensory ataxia indicated by a previous report [4].

\section{Conclusions}

We and others are proposing that a pure thalamic infarct can be the trigger for a posterior ALS variant [19]. Our case indicates that the onset can be acute and associated with movement disorders that need to be quickly recognized. This ALS variant posits the necessity of a careful differential diagnosis with other movement disorders (i.e., hemiballism, chorea, pseudoathetosis, mirror movements, focal seizures) as the condition appears to be self-containing, transient in nature and tends to disappear or improve with time [2] but nevertheless require therapeutic interventions targeted at ongoing cerebrovascular conditions.

\section{Statement of Ethics}

The patient's written informed consent was obtained for publication of medical records, including images. 


\section{Disclosure Statement}

The authors declare that the research was conducted in the absence of any commercial or financial relationships that could be construed as a potential conflict of interest. This study is not industry-sponsored.

\section{Funding Sources}

No targeted funding reported.

\section{Author Contributions}

M.R. drafted the manuscript. M.R., C.C., F.D., M.V.D.A., and V.D.S. managed the patient and collected clinical data. S.L.S. and M.O. revised the manuscript and edited the paper.

\section{References}

1 Goldstein K. Zur Lehre von der motorischen Apraxie. J Psychol Neurol. 1908;11:169-87.

2 Hassan A, Josephs KA. Alien Hand Syndrome. Curr Neurol Neurosci Rep. 2016 Aug;16(8):73.

3 SPREAD - Stroke Prevention and Educational Awareness Diffusion. Ictus cerebrale: Linee guida italiane di prevenzione e trattamento [cited 2016 July 21]. Available from: http://www.isospread.it/capitoli/LINEE_GUIDA_SPREAD_8a_EDIZIONE.pdf.

4 Marey-Lopez J, Rubio-Nazabal E, Alonso-Magdalena L, Lopez-Facal S. Posterior alien hand syndrome after a right thalamic infarct. J Neurol Neurosurg Psychiatry. 2002 Oct;73(4):447-9.

5 Panikkath R, Panikkath D, Mojumder D, Nugent K. The alien hand syndrome. Proc Bayl Univ Med Cent. 2014 Jul;27(3):219-20.

6 Spector AR, Freeman WD, Cheshire WP. The stroke that struck back: an unusual alien hand presentation. J Stroke Cerebrovasc Dis. 2009 Jan;18(1):72-3.

7 Yuan JL, Wang SK, Guo XJ, Hu WL. Acute infarct of the corpus callosum presenting as alien hand syndrome: evidence of diffusion weighted imaging and magnetic resonance angiography. BMC Neurol. 2011 Nov;11(1):142.

8 Pack BC, Stewart KJ, Diamond PT, Gale SD. Posterior-variant alien hand syndrome: clinical features and response to rehabilitation. Disabil Rehabil. 2002 Oct;24(15):817-8.

9 Ventura MG, Goldman S, Hildebrand J. Alien hand syndrome without a corpus callosum lesion. J Neurol Neurosurg Psychiatry. 1995 Jun;58(6):735-7.

10 Graff-Radford J, Rubin MN, Jones DT, Aksamit AJ, Ahlskog JE, Knopman DS, et al. The alien limb phenomenon J Neurol. 2013 Jul;260(7):1880-8.

11 Bartolo M, Zucchella C, Pichiecchio A, Pucci E, Sandrini G, Sinforiani E. Alien hand syndrome in left posterior stroke. Neurol Sci. 2011 Jun;32(3):483-6.

12 Kato H, Iijima M, Hiroi A, Kubo M, Uchigata M. [A case of alien hand syndrome after right posterior cerebral artery territory infarction]. Rinsho Shinkeigaku. 2003 Aug;43(8):487-90.

13 Levine DN, Rinn WE. Opticosensory ataxia and alien hand syndrome after posterior cerebral artery territory infarction. Neurology. 1986 Aug;36(8):1094-7.

14 Groom KN, Ng WK, Kevorkian CG, Levy JK. Ego-syntonic alien hand syndrome after right posterior cerebral artery stroke. Arch Phys Med Rehabil. 1999 Feb;80(2):162-5.

15 Doody RS, Jankovic J. The alien hand and related signs. J Neurol Neurosurg Psychiatry. 1992 Sep;55(9):80610.

16 Kim JS, Caplan LR. Clinical Stroke Syndromes. Intracranial Atherosclerosis: Pathophysiology, Diagnosis and Treatment, Front Neurol Neurosci. Volume 40. Basel: Karger; 2016. pp. 72-92.

17 Gupta N, Pandey S. Post-Thalamic Stroke Movement Disorders: A Systematic Review. Eur Neurol. 2018;79(56):303-14.

18 Ay H, Buonanno FS, Price BH, Le DA, Koroshetz WJ. Sensory alien hand syndrome: case report and review of the literature. J Neurol Neurosurg Psychiatry. 1998 Sep;65(3):366-9. 


\section{Case Reports in Neurology}

\begin{tabular}{l|l}
\hline Case Rep Neurol 2020;12:35-39 \\
\hline DOI: 10.1159/000503857 & $\begin{array}{l}\text { c 2020 The Author(s). Published by S. Karger AG, Basel } \\
\text { www.karger.com/crn }\end{array}$ \\
\hline
\end{tabular}

19 Moro V, Pernigo S, Scandola M, Mainente M, Avesani R, Aglioti SM. Contextual bottom-up and implicit topdown modulation of anarchic hand syndrome: A single-case report and a review of the literature. Neuropsychologia. 2015 Nov;78:122-9.

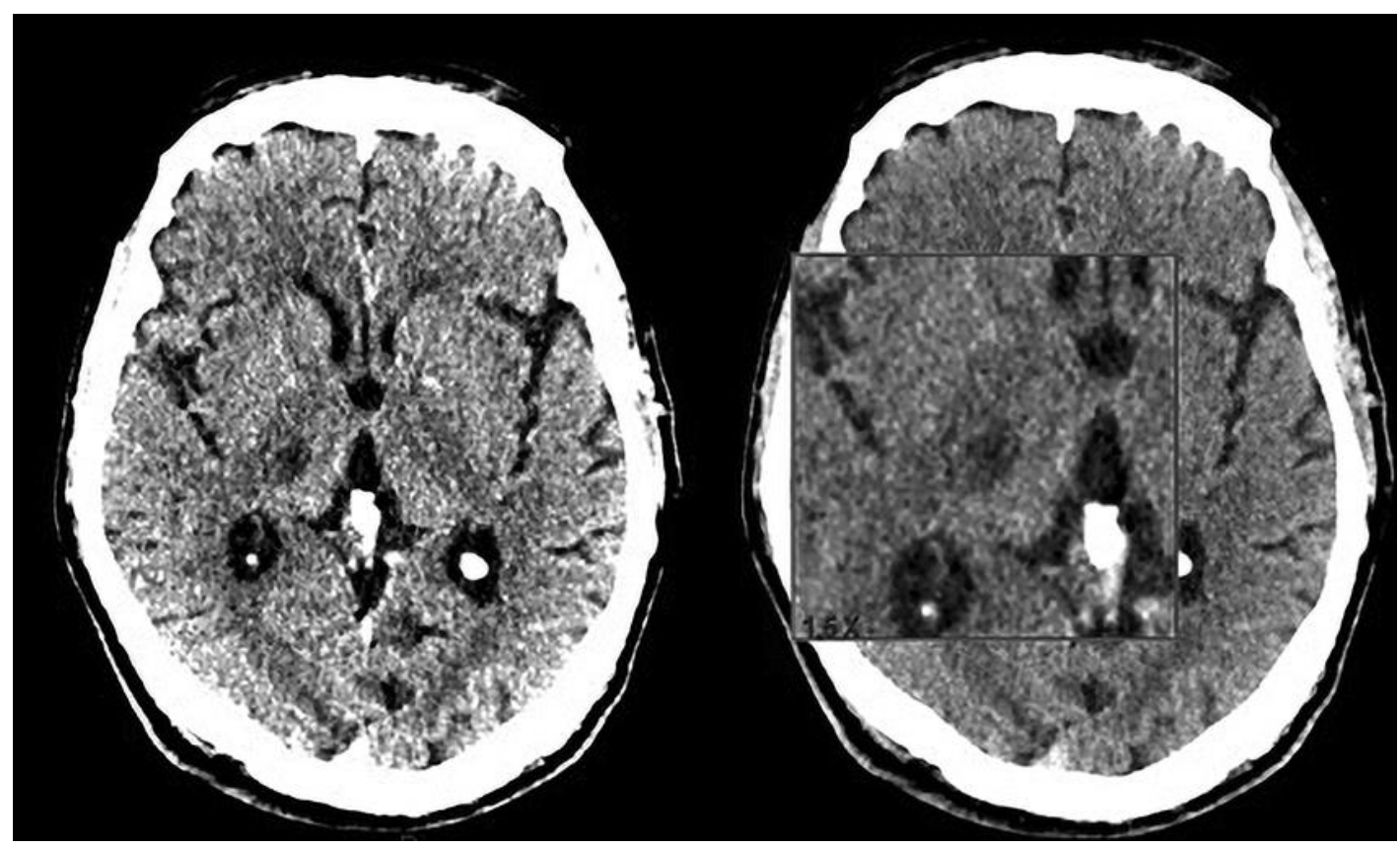

Fig. 1. 24-h computed tomography brain scan showing a new-onset right thalamic ischaemic lesion. 